\title{
The Economic War Between USA and China and Future Scenarios
}

\author{
Islam Mohamed Mahmoud*, Marwan Waheed Abdel Hamid \\ Department of Banking and Financial Sciences, Al-Maaref University College, Anbar, Iraq \\ * islambekhet84@oua.edu.iq
}

\begin{abstract}
:
With the expansion of foreign trade and the burning of competition between countries, the value of currencies began to play an effective and effective role in the global economy, as some countries resorted to reducing the exchange rates of their currencies against the currencies of other countries in order to make their exports more competitive, and the manifestations of the conflict between America and China over the prices of Exchange their currencies, as America accuses China of interfering in exchange and evaluating the yuan under its real value. While the Chinese view the expansionary and extremist monetary policies of the Federal Reserve as an act of currency manipulation, some fear that this conflict will develop into a competitive reduction race that could cause global trade shrinkage and stagnation, and undermine confidence internationally in the international monetary system, which It causes a global financial and economic crisis with a profound negative impact on the entire global economy and the developing economies in particular, which some believe will be the biggest loser of that conflict. The relationship between Washington and Beijing is currently witnessing a wave of political and strategic escalation, especially from the American side, and despite this this the escalation is not new from its predecessors during the past period. But in terms of form, it may be the most severe, but it will not reach the war. Both countries need each other, whereas as much as there are tensions and fundamental differences, it is matched by the existence of common interests.
\end{abstract}

Keywords: Economic War; Devaluation; Currency Wars; China; USA. 


\section{الحرب الاقتصادية بين أمريكا و الصين و السيناريوهات المستقبلية \\ م.م. اسلام محمد محمود"، م.م. مروان وحيد عبد الحميد \\ قسم العلوم المالية والمصرفية،كلية المعارف الجامعة، الانبار، العر اق \\ * islambekhet84@oua.edu.iq}

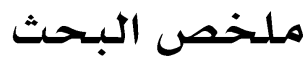

مع توسع التجارة الخارجية واشتعال المنافسة بين الدول بدأت قيمة العملات تلعب دوراً مؤثراً وفاعلاً في الاقتصاد العالمي، حيث لجأت بعض الدول الى تخفيض أسعار صرف عملاتها مقابل عملات الدول الأخرى بغرض جعل صادراتها اكثر تنافسية، وقد اتسعت في الآونة الأخيرة مظاهر الصراع بين امريكا والصين حول أسعار صرف عملتيها حيث تتهم امريكا الصين بتدخلها في الصرف وتقييمها لليوان بأقل من قيمته الحقيقية، في حين ينظر الصينيون الى السياسات النقدية التوسعية والمتطرفة للاحتياطي الاتحادي على أنها عمل من اعمال التلاعب بالعملة، ويتخوف البعض من إمكانية ان يتطور هذا الصراع الى سباق التخفيض التنافسي وهو ما يمكن ان يتسبب في انكماش التجارة العالمية والركود، وزعزعة الثقة دولياً في النظام النقدي الدولي مما يسبب ازمة مالية واقتصادية عالمية ذات تأثير سلبي عميق على الاقتصاد العالمي برمته والاقتصادات النامية بشكل خاص، والتي يرى البعض انها ستكون الخاسر الأكبر من ذلك الصراع، وتشهد العلاقة بين واشنطن وبكين حالياً موجة من التصعيد السياسي والاستراتيجي خاصة من الجانب الامريكي وعلى الرغم من ذلك فان هذا التصعيد ليس بجديد عن سابقاته خلال الفترة المنصرمة الماضية، لكن من حيث الشكل قد يكون الاكثر حدة لكنه لن يصل الى الحرب فكلا الدولتين تحتاجان الى بعضها، حيث بقدر وجود التوترات والخلافات الجحوهرية فانه يقابلها وجود مصالح مشتركة.

الكلمـات المفتاحيـة: الحرب الاقتصاديـة، تخفيض قيمـة العملة، حروب العملة، الصين،

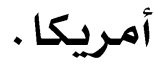




\section{المقدمة}

شهد الاقتصاد العالمي المعاصر والنظام النقدي الدولي تحولات جوهرية عميقة تجلت بسلسة من المظاهر ابرزها، فك ارتباط الدولار بالذهب عام 1971 كتعبير عن ازمة الاقتصاد الأمريكي التي رافقت ادائه منذ عقد الستينيات من القرن الماضي نتيجة تنامي عجزه المزدوج المتمثل في عجز ميزان المدفوعات وعجز الموازنة العامة (دانيالز،12 2016، 116)، وذلك بسبب الانفاق الهائل الذي كانت ولازالت تموله الولايات المتحدة بطبع المزيد من مليارات الدولارات دون غطاء حقيقي في اطار ما يسمى بسياسة التيسير الكمي (QE)، مستفيدة من وضع الدولار كعملة مهيمنة على نظام النقد الدولي والتي تعد احدى وسائل الحرب الاقتصادية. وكذلك قيام الاتحاد الاقتصادي والنقدي الاوروبي والذي اصبحت عملته الموحدة (اليورو) تمثل ثاني أكبر العملات نصيباً من اجمالي الاحتياطيات العالمية وتمثل تهديدا كبيرا لمكانة الدولار المهيمن على النظام النقدي الدولي اضافة الى الازمة الاقتصادية والمالية العالمية عام 2008 والتي بدأت بالاقتصاد الامريكي وسرعان ما انتشرت في معظم الاقتصادات الاوروبية وكذلك الاقتصادات المرتبطة بهاذين الاقتصادين بصورة مباشرة، وبكافة الاقتصادات الاخرى بصورة غير مباشرة. وشهلت الساحة الاقتصادية العالمية ظهور اقتصادات ناشئة او ناهضة عملاقة مثل الاقتصاد الصيني والروسي والهندي والكوري والبرازيلي، والتي اصبح بعضها ينافس الاقتصادات المتقدمة والبعض الآخر أكثر تنافسية منها خاصة الاقتصاد الصيني الذي يمثل ثاني اكبر اقتصاد في العالم بعد الاقتصاد الأمريكي، واكبر اقتصاد يدير فائض تجاري مع الولايات المتحدة، و اكبر دائن فضلا عن قيام بعقد عدد من اتفاقيات مبادلة العملة مع عدد من الدول سعيا لتجاوز الدولار في مبادلاتها الثنائية، فضلا عن مساعيها الحثيثة لتدويل الرنمينبي (Murphy، 2009، 12-13)، ودعواتها المتكررة لإصلاح النظام النقدي الدولي بالتنسيق مع بعض دول منطقة اليورو خاصة فرنسا. 
هذه العوامل وغيرها اصبحت تهدد ويقوة سيطرة الاقتصاد الامريكي على النظام الاقتصادي العالمي وهيمنة الدولار على النظام النقدي الدولي، مما يوحي حسب رأي بعض المحللين بانهيار النظام النقدي الدولي الحالي وانبثاق نظام اقتصادي ونقدي عالمي جديد في المستقبل المنظور (ضمد، 2012، 5).

\section{أهميـة ومشكلة البحثث:}

قامت امريكا بشأن ما يسمى (الحرب الاقتصادية) كمحاولة منها لتجاوز تداعيات الأزمة الاقتصادية ولأنعاش اقتصادها، وتعزيز تنافسيتها، متهمة غيرها بالبدء بهذه التنافس وخاصة الصين، ورغم أن تاريخها الاقتصادي القريب كما يرى بعض المحللين يؤكد استخدامها لسياسة تخفيض سعر الصرف وهي جوهر الحرب الاقتصادية لتحقيق مكاسب اقتصادية وتجارية على حساب الدول الأخرى، فضلا عن قيامها سابقا بفك ارتباطه بالذهب رغم التزامها بذلك وفق اتفاقية بريتون وودز (ضمد، 2012، 6 ). ورغم ان البعض يرجع تاريخ الحرب الاقتصادية الى ازمة الكساد العظيم ( 1929_1933 ) كسبب ونتيجة لهذه الأزمة، فان البعض يؤكد ان هذا المصطلح ظهر لأول مرة على لسان وزير المالية البرازيلي في سبتمبر 2010، نتيجة الصراع الدائر بين أكبر اقتصادين في العالم (الاقتصاد الامريكي ونظيره الصيني). فمن وجهة نظر السياسة الصينين فان السياسات النقدية التوسعية والمتطرفة للاحتياطي الاتحادي والتي تهدف المى خفض قيمة الدولار بهدف زيادة تنافسية الصادرات الامريكية، هي عمل من الاعمال التلاعب بالعملة (جلال، 2014 ). وفي هذا السيناريو الخطير للتنافس الاقتصادي والتي يمكن ان ينتهي بها المطاف الى حمائية تجارية، فإن مواقف طرفيها تبدو متعارضة، فبينما تطالب الولايات المتحدة بتعويم الرنمينبى (اليوان)، فان الصين تطالب بإعادة التفاوض على وضع الدولار كعملة دولية رئيسية وانشاء نظام نقدي دولي أكثر استقرارا وتنسيقا .(3،2011،Miguel)

\section{فرضية البـحث:}

يفترض البحث ان الحرب الاقتصادية لها تأثيرات متباينة في النظام النقدي الدولي والدول النامية. 


\section{منهجية وهيكلية البحثث:}

اعتمد الباحثان على المنهج الاستقرائي الوصفي وسعياً لتحقيق الاهداف المتوخاة من هذا البحث تم تقسيمه

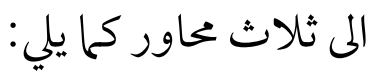
المحور الاول: مظاهر الحرب الاقتصادية بين الولايات المتحدة والصين وموقف الاتحاد الأوربي. المحور الثاني: الافاق المستقبلية للدولار في نظام النقد الدولي في ظل صراع الحرب الاقتصادية. المحور الثالث: السيناريوهات المستقبلية للتنافس الاقتصادي بين أمريكا والصين.

\section{المحور الأول: الحرب الاقتصاديـة بين امريكا والصين وموقف الاتحاد الأوربي يتناول هذا المحور مفهوم الحرب الاقتصادية وأدواتها ومظاهر ها بين امريكا والصين.}

\section{أولاً: : المفهوم}

يقصد بالحرب الاقتصادية اعتماد دولة ما على قوتها الاقتصادية لتعزيز قوتها التنافسية وتقليص قوة تنافسية الدول الاخرى ومن ثم تقليص حجم ثروتها، وذلك بالتدخل في سوق الصرف الأجنبي (الفوركس) ليعد شكلا من اشكال الحرب الاقتصادية الباردة (جلال، 2010، 13)، (الفقيه، 2011، 11)، (بن غيث، 2011، 33 3)، كذلك يعر فها آخرون بأنها لا تعدو كونها تخفيض تنافسي لأسعار صرف عملات الدول الفعالة في الاقتصاد الدولي لتحقيق مكاسب محتملة على حساب بعضها البعض (ضمد، 2012، 8)، من هنا أطلق البعض على هذه الظاهرة " سياسة افقار الغير(كريانين، 10 20، 84 3)، وترى (أليس روز) خبيرة العملات بمجموعة جي بي مورغان لإدارة الاصول " ان الحرب الاقتصادية سببها تحديد قيمة العملة بعيدا عن قو اعد السوق (محمود، 2013، 13 13، كما وصفها الصحافي الصيني لوشياو بو بانها حرب بدون دخان (Murphy)

$$
\text { 2009، 600 ) كما تعرف ايضاً بحروب العملة. }
$$




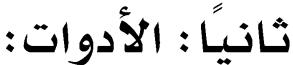

تمتلك الحكومات ومصارفها المركزية وسائل متعددة يمكنها من خلالها التدخل لتخفيض اسعار صرف عملتها الوطنية مقابل عملات الدول الاخرى خاصة العملات الرئيسية ويمكن تقسيمها الى: 1. 1 تدخلات مباشرة واهمها (جلال، 10 201 14): قيام البنك المركزي بشر اء العملة المحلية وبيع العملة الأجنبي. قيام البنك المركزي بتخفيض سعر الفائدة، ومن ثم انخفاض الطلب على العملة المحلية نتيجة انخفاض العائد عليها مايؤدي الى انخفاضها فيما يعرف بسياسة التيسير الانتهائي (Credit Easing) الا ان هذا الاجر اء محكوم ايضا بحالة الاقتصاد القائم واولوياته. 2. تدخلات غير مباشرة واهمها (بن غيث، 2011، 3 9):

زيادة المعروض النقدي من العملة المحلية في النظام المصرفي فيا يعرف بسياسة التيسير الكمي (Quantitative Easing)، وقد استخدمت هذه السياسة على نطاق واسع ابان الازمة المالية الاخيرة، خاصة من قبل بنك الاحتياطي الفيدرالي الامريكي فبعد الازمة الاخيرة قام البنك بضخ مبلغ كبير من الدولارات للسوق فيها عرف بالجولة الثانية من التيسير الكمي بلغت (60) مليار دو لار. ضبط حركة رؤوس الاموال (capital control) وذلك بفرض قيود على حجم تحويلات النقدية، او تحويلات العملة المسموح بها (Exchange control)، او فرض ضريبة على معاملات تحويل ( Exchange) Tax ). وعادة ما تلجأ الحكومات الم انتهاج سياسة التيسير الكمي كأجراء اخير بعد استنفاد كل خيارات السياسة النقدية (بن غيث، 2011، 33 ).

\section{ثالثًا : مظاهر الحرب الاقتصادية بين الولايات المتحدة والصين:} اتسعت في الآونة الاخيرة مظاهر الصراع بين امريكا والصين حول اسعار صرف عمليتيها والسياسات المتخذة بشأنها، اذ تتهم الولايات المتحدة الصين بأنها تقوم بتخفيض قيمة اليوان مقابل الدولار الامريكي بوسائل متعددة، سواء من خلال قيامها بشراء العملات الأجنبية (وفي مقدمتها الدولار) مما تسبب في زيادة 
عرض اليوان الصيني ومن ثم انخفاض سعر اليوان مقابل الدولار، او من خلال تدخل المؤسسات المالية و النقدية الصينية باتخاذ التدابير التي تحول دون ارتفاع قيمة اليو ان رغم زيادة فائض الميزان التجاري الصيني، الذي يفترض ان يقترن بارتفاع قيمة اليوان وتطالبها بتعويم اليو ان (العبود، www.aliraqi.org)، في حين ينظر الصينيون المى السياسة النقدية الأميركية باعتبارها عمل من اعمال التلاعب بالعملة، ويطالبون بإعادة التفاوض على وضع الدولار كعملة دولية رئيسية وانشاء نظام سعر صرف دولي أكثر استقرار وتنسيقاً (Miguel، 2011،

وتدعي الو لايات المتحدة ان زيادة عجزها التجاري مقابل زيادة الفائض الصيني يرجع لانخفاض قيمة اليوان بفعل السياسات الصينية، مما اكسب الصادرات الصينية ميزة تنافسية مقابل شركائها بالتجارة واهمهم الولايات المتحدة، فيما يعرب الصينيون عن مخاوفهم بشأن السياسة المالية التوسعية لإدارة اوباما وتبعات التمويل بالعجز الذي تنتهجه الولايات المتحدة في اعقاب الازمة المالية العالمية (Murphy، 2009، 2)، (مين شين بيه، 2010) ، وقد حاولت الو لايات المتحدة الضغط على الصين بشتى الوسائل، لرفع قيمة اليوان لكن الصين لم تخضع لتلك الضغوطات معتبرة ان ضعف اليو ان يساهم في زيادة صادر اتها التي غزت العالم، والتي تشكل عصب الاقتصاد الصيني نظر ال انتاجها سياسة النمو القائم على التصدير (عادل، jef1.kcorp.net). ومن اهم مظاهر الحرب الاقتصادية بين الصين والولايات المتحدة ما يلي: 1 - دعوات الصين المتكررة لإصلاح النظام النقدي الدولي الحالي والتخلص من هيمنة الدولار، حيث يتذمر المسؤولون الصينيون من الامتياز الباهظ الذي تملكه الولايات المتحدة بإصدار العملة الدولية الرئيسية، مشيرين المى عدم الاستقرار المصاحب لهذا الوضع. ويقترحون، كما كان الحال في اواخر الستينيات مع الفرنسين، زيادة استخدام حقوق السحب الخاصة لصندوق النقد الدولي (SDR) وهي شبع عملة تستخدم بواسطة صندوق النقد الدولي، يكون استخدامها كعملة احتياط عالمية (Miguel، 2011، 2)، وقد نشر محافظ البنك المركزي الصيني ثلاثة مقالات تدعوا المى اصلاح النظام المالي العالمي، محذرا 
الو لايات المتحدة بشأن سياساتها النقدية والمالية التوسعية، ومطالبا بالأشر اف عن قرب على تلك البلدان

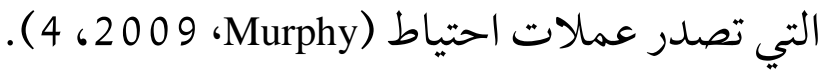

2- تحرك الصين باتجاه تنويع الاحتياطي، استطاع الصينيون مقاومة السياسة النقدية الامريكية التوسعية

بفضل ما تراكم لديها من احتياط دو لاري ضخم يبلغ (2.85 ) تريليون دولار، فمع هذا المبلغ الضخم من الاحتياطات، وما يقارب (55\%) منها مقوما بالدولار الامريكي - اكتسبت الصين حماية استقلالية وقدرة اعلى للتصدي لأي هجوم مضارية يأتي من المؤسسات المالية التي تعمل انطلاقا من وول ستريت. لهذا رغم محاولات الولايات المتحدة من خلال آليات " الحرب الاقتصادية " (اي التيسير الكمي) تخفيض قيمة الدولار ورفع قيمة الرنمينبي، فإن الصين قادرة على المقاومة بفضل ما لديها من احتياطي دولاري (Miguel، 2011، 3)، وللمزيد من التنويع، صرح نائب محافظ البنك المركزي ورئيس ادارة النقد الأجنى الصيني بأن الصين فد غززت احتياطاتها الذهبية لتبلغ (1054) طناً، مما جعل الصين خامس أكبر دولة في العالم امتلاكا للذهب (الصين اليومية، 11 / 5 / 2009)، كما افيد ايضا بان مكتب الاحتياطي للدولة يخطط لشراء الالمنيوم، والنحاس، والزنك، والرصاص (Murphy، 200 20 7-8 ). كما ان الصين كانت مستعدة لشراء ما يصل الى ( 0 ) بليون من السندات الصادرة عن صندوق النقد الدولي، وهذه السندات سنكون مقومة بحقوق السحب الخاصة (SDRs)، وقد دقت اجراس الإنذار عندما اظهرت وزارة الخزانة الامريكية بان بكين تمتلك 763.5 بليون دولار من السندات الامريكية نزولا من (9.9. 767 ) بليون دولار خلال شهر واحد، وهو اول اقتطاع في موجوداتها الاجمالية منذ فبراير عام Murphy) 2008 2009، 8). ويرى البعض ان الصين بامتلاكها هذه الاحتياطات الهائلة من الدولارات الامريكية قد حققت توازن رعب اقتصادي بينها وبين الولايات المتحدة، اذ تستطيع في حالة ضخها هذا الرصيد الهائل من احتياطاتها الدولارية في الاسواق العالمية الاطاحة بعرش الدولار المهتز اصلا، الامر الذي قد يعجل بنهاية هيمنته على النظام النقدي الدولي، ولذا لم تستطيع الإدارة الامريكية 
من الناحية الواقعية عمل شيء ملموس في إطار الحرب الاقتصادية مع الصين رغم تهديدها المستمر بفرض عقوبات اقتصادية ورسوم جمركية على السلع الصينية (ضمد، 12012 20 7 ). وعلى الرغم من ان اي تنوع في احتياطاتها في المدى القصير الى المتوسط فان الحكومة الصينية لديها لجوء قليل في مو اصلة الاستثمار في سندات الخزانة نظر العدم وجود اسواق مالية اخرى عميقة بها يكفي ولديها السيولة بها يكفي للتعامل مع الاستثمارات الصينية الضخمة (Murphy، 2009، 8 2). 3- مساعي الصين لتدويل الريمنبي، حيث يبحث صناع القرار الصينيين في كيفية تمكن اليورو ان يصبح عمله دولي في اقل من عقد وانهم في محاكاة هذا المسار الناجح مع الرنمينبى (Miguel، 2011، 10)، ومنذ أواخر عام 2008 اتخذت الصين خطوات لتدويل اليوان الريمنبي، بها في ذلك توسع في استخدامه في التسويات عبر الحدود والتجارة الدولية وعقد سلسلة من الاتفاقيات المربحة التبادل العملات

.(19،2011،Dadush)

وعلى خليفة الازمة المالية العالمية، بدأت الصين حملة متعددة المراحل لزيادة الاستخدام الرنمينبى في التجارة الدولية ، كما كشف احد المسؤولين الصينيين، ان الدروس المستفادة من عملية تدويل اليورو توفر نموذجا جديدا عن كيفية تطوير هذه الاستراتيجية بنجاح، كما بدأ بجلس الدولة في خطوة كبيرة نحو تطوير سوق العملات الخارجي ودعم تدويل الرنمينبى بالسماح بتسجيل المؤسسات المالية في هونغ كونغ لا صدار سندات مقومة الرنمينبى، كما أجرى بنك الصين الشعبي مناقشات مع سنغافورة وذلك لا نشاء المركز الثاني لتجارة الرنمينبى بالخارج (Miguel، وفي يناير 2011 اعلنت الصين مبادرات فردية كبيرة لتدويل الرنمينبى وتم السماح للشركات الصينية بنقل الرنمينبى خارج الصين لأجل الاستثمار بالخارج كذلك السماح للبنوك الصينية بالتوسع في قروض الرنمينبى لهذا الغرض، واي ارباح من هذه الاستثمارات يمكن اعادتها الى الرنمينبى (Dadush، 2011، 8 6)، ومقارنة بالسرعة السابقة التي صعد بها فجأة الدولار الامريكي كعملة مهيمنة عالميا بعد 1913، 
يتوقع البعض ان تدويل الرنمينبى كما يمكن ان يكون بنفس السرعة تماما، وهناك شعور عام متفائل بين النخب المالية الصينية عن المستقبل الرنمينبى في النظام النقدي الدولي (IMS). 4- قيام الصين بتوقيع عدد من اتفاقيات مبادلة العملة، في تحدي واضح لهيمنة الدولار، بدأت الصين جهودها بعقد عدد من اتفاقيات مبادلة العملة الثنائية في 2008 مع كوريا الجنوبية، ماليزيا، بيلاروس، اندونيسيا، الارجنتين، ايسلندا، وسنغافورا، وذلك لإلغاء الدولار من معاملات التجارة الثنائية، وفي يناير 2009 وقعت اتفاقيات مماثلة إضافية مع هونغ كونغ، ومع نيوزيلاند واوزبكستان في ابريل 2011، ومع روسيا في يونيو 2011، كما ان الصين تناقش استخدام العملات المحلية لتسوية التجارة مع البرازيل حيث توصلوا الم تفاهم مبدئي لإلغاء الدولار تدريييا في التجارة الثنائية (Dadush، 2011، 20 )، ويشكل مماثل هناك اتفاق للتحرك باتجاه تسوية التجارة البينية بعملة كل منها قد تم التوصيل اليه بين بكين وموسكو (Murphy)، ومنذ قمة مجموعة العشرين في نوفمبر عام 2008، وقع بنك الصين الشعبي (PBOC) اتفاقيات مبادلة عملة ثنائية بأجمالي (650) بليون RMB (5 65 بليون دولار)، وفي عام 2011 قدرت اتفاقيات المبادلة (829) بليون RMB حوالي (130) بليون دولار (Dadush، 2011، 20)، وطبقا لبعض المحللين فأن أحد الاهداف الرئيسية للصين من بدء الاتفاقيات مبادلة عملتها اضافة الى تجاوز الدولار، هو تشجع التعاون المالي الاقليمي وتعزيز قدرة الصين على توفير السيولة المساعدة للأخرين (Murphy، 2009، 12)، وبينما ينظر الصينيون المى مقايضات العملة على انها طريقة جيدة لتنويع احتياطيات الصين الدولارية الضخمة، فإن لبعض المراقبين الأجانب، وأن المقايضات هي دليل آخر على ان "الصينين في مسار لا بأس فيه تجاه تحدى الدولار (Murphy، 2009، 14)، ومع ذلك ارجع البعض ان اتفاقيات مقايضة العملات ترجع في جزء منها، لأسباب سياسية (Dadush، 2011، 22)، حيث المبررات الاقتصادية اقل وضوحا وان الدوافع الجيوسياسية للصين تظهر جلياً (Murphy، 2009، 13) وهنا تتجلى خطورة هذا الصراع. 
5- موقف الاتحاد الاوروبي من الصراع الامريكي الصيني، حيث تحتاج الصين ايجاد تحالفات في مساعيها لا صلاح النظام النقدي الدولي والتخلص من هيمنة الدولار الامريكي، وأفضل حليف سيكون الاتحاد الأوربي (EU)، وهذا هو السبب الذي جعل الصين تتطلع الى اوروبا لتساعدها في ادراج اصلاح النظام النقدي الدولي (IMS) على جدول اعمال قمة بجموعة العشرين (G20)، ويعول بعض المسؤولين الصينين بشكل خاص على التعاون الفرنسي في هذا الصدد باعتقادهم إن الفرنسين متحمسين للابتعاد عن معيار

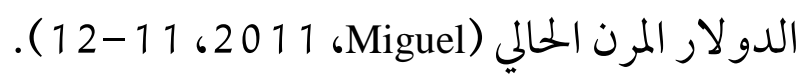
ويدرك الصينيون ان الخبرة الاوربية في المفاوضات المتعددة الاطراف هي اساس معرفي جيد لدفع اصلاح النظام النقدي الذي يتسم بهيمنة الدولار قدما، وقد أكد أحد الباحثين في الأكاديمية الصينية للعلوم الاجتماعية (CASS)، انه ولعدة سنو ات كان احد العاملين بالبنك المركزي الايطالي يتحدث كثيرا عن حقوق السحب الخاصة مع الباحثين في الاكاديمية، وقد اجرى مؤخرا اتصالا مع البنك المركزي الصيني لشرح الاقتراح، كما ان روبرت منديل الذي كانت له مساهمة مؤثرة في خلق اليورو قد اوصى الصين بإصلاح نظام النقد الدولي ( Miguel، 2011، 13 ) . وقد شجع نجاح اليورو وخبرة الاوربيين ودرايتهم بالتعددية النقدية صناع القرار بالصين ليطالبوا الاوربيون ليذهبوا ابعد من ذلك والبدء بتعاون نشط مع الصين في الجهود المبذولة لأنشاء نظام اقتصادي أكثر توازن وعدالة، ذلك على الاقل سيشكل ضغطا على الولايات المتحدة لتكون أكثر حذرا مع سياساتها النقدية، وبوجه عام استجاب الاوربيون بحرارة لدعوات الصينيين من اجل انهاء معيار الدولار المرن (FDS)، ورغم ذلك عندما نشر (تشو تشو شياو تشوان) مقالة عن اصلاح النظام النقدي الدولي عام 2009، فان كل الاسواق الناشئة الاخرى والعديد من خبراء النظام النقدي الدولي قد اطرتهم الفكرة، الا ان رد الاوربيين كان باهتا، لهذا، وبالرغم من هذه المناوشات الدبلوماسية، فانه حتى عام 2010 كان دور اوروبا في اصلاح النظام النقدي الدولي خيبا للآمال وذلك من جهة نظر النخب المالية في الصين، وتشير استطلاعات الراي ان الغالبية العظمى من الصينيين يودون ان يروا 
اوروبا أكثر استقلالا وحزما، وايضا أكثر في مساعدة الصين لإصلاح النظام النقدي الدولي (IMS) والتي يمكن ان تكون بمثابة توازن مضاد للو لايات المتحدة، ويقول بعض المسئولين الصينيين ان الاوربيين يملكون حق النقض بسبب نظام التصويت، ولكن بترك نظام التصويت جانبا، فان الحقيقة هي ان صندوق النقد الدولي (IMF) تتحكم به الو لايات المتحدة تماما، اما بالنسبة للأوروبيين، فهم يعينون المدير العام، لكن هذا فقط لحفظ ماء وجوههم، وهم بحاجة لبذل المزيد من الجهود لو أرادوا تغيير الواقع (15،2011،Miguel) وعلى الرغم من ان الأوروبيين ككتلة موحدة لا يخرجون بموقف واضح تجاه دعوة الصين لا صلاح نظام النقد الدولي (IMS)، الا ان الحكومة لها نهج مختلف، فني يوليو عام 2009 في اجتحلع بجموعة العشرين (G20) في ايطاليا، دعم الرئيس ساركوزي المحاولات الصينية لإصلاح معيار الدولار المرن بقوله (اننا لا نستطيع ان نلتصق بعملة وحيدة) وينبغي ان يتو افق عالم متعدد الاقطار سياسيا مع عالم متعدد العملات اقتصاديا، وبعد ستة أشهر من المنتدى في دافوس أعلن الرئيس الفرنسي " لا يمكن ان على حرية التجارة ونتساهل مع الاغراق النقدي" في اشارة واضحة الى سياسة التيسير الكمي التي تنتهجها الو لايات المتحدة "، وبدا الفرنسيون معنيون بدفع اصلاح نظام النقد الدولي (IMS) على جدول اعهال قمة بجموعة العشرين، لانهم كانوا يعرفون ان بإمكانهم الاعتماد على الدعم الصيني (Miguel، .$(17$ ، 2011 الا انه وبالرغم من ان الاوربيين من خلال الاتحاد الاقتصادي والنقدي (EMU) وتعزيز اليورو قد اكتسبو ا استقلال ذاتيا وتزودوا ببعض النقود بها يتعلق بالمظهر الثاني من النفوذ في شكل نشر الافكار عن البدائل الممكنة لمعيار الدولار المرن (FDS) وتشكيل جدول الاعمار لا صلاحه، فانهم لايزالون بعيدين عن تشكيل اي تأثير مهم في المظهر الاول حيث تقرر في الواقع قواعد اللعبة للنظام النقدي العالمي، والسبب الرئيسي لذلك انهم لا يملكون وحدة سياسية ليتجاوزوا المظهر الثاني وهذا بالتأكيد يمثل 
عائقا في بناء دبلو ماسيا نقدية اوربية موحدة تكون قادرة على موازنة النفوذ النقدي للولايات المتحدة (20،2011،Miguel)

\section{المحور الثاني: الآفاق المستقبلية للدولار يخ نظام النقد الدولي يِ ظل الحرب الاقتصادية} على غرار ما حدث في نهاية الستينيات، عندما انهار نظام بيتيون وودز، وفي الثثانينات، عندما كانت العجوزات الثنائية من الولايات المتحدة تتصاعد، وفي الآونة الاخيرة (خاصة بعد " انهيار وول ستريت"، في 2008)، أصبح هناك نقاش متز ايد بين علماء الاقتصاد السياسي الدولي (IPE) بشأن ما إذا كانت هيمنة الدولار في النظام النقدي الدولي موضوع شك، والآن هناك متغيرات جديدة تجعل هذا النقاش مختلف، حيث في الماضي كان جيسكار ديستان وشارل ديجول يشكوان من "الامتياز الباهظ" الذي تملكه الولايات المتحدة بإصدار العملة الدولية الرئيسية، واليوم يوجد (تشوشياو تشوان) محافظ البنك المركزي الصيني و(هوجين تاو) رئيس الصين، واللذان يذكران انتقادات مماثلة، مشيرين الى عدم الاستقرار المصاحب لـ(معضلة تريفين) ويقترحان كما كان الحال في اواخر الستينيات مع الفرنسين زيادة استخدام حقوق السحب الخاصة لصندوق النقد الدولي لتكون هي العملة الدولية بامتياز بدلا من الدولار الامريكي، ومع توحيد اليورو واصرار الصين على اصلاح النظام النقدي الدولي الحالي تواجه العملة الرئيسية للنظام النقدي الدولي الحالي تواجه العملة الرئيسية للنظام النقدي الدولي (الدولار) منافسة أكثر صرامة بشان العملة المهيمنة، ويقول موردخاي كريانين ان النمو المتسارع لبعض الاقتصادات الاخرى سيؤدي المى تغير ميزان القوى للنظام الاقتصادي العالمي وتحويله من احادي القطب (الولايات المتحدة) الم النظام متعدد الاقطاب تقوده آسيا وأوروبا والولايات المتحدة

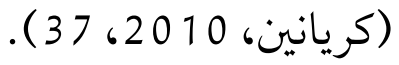
الا ان البعض يرى ان النقاش الدائر حاليا حول زو ال هيمنة الدولار يبدو مبالغا فيه الى حد ما، وان الدعوات المطالبة بالبديل ماز الت موجودة، لكنها لم تتجسد بعد، فبالرغم من ان الدولار لم يعد المرسى او المرجعية لربط كل العملات، الا انه يبقى عملة الاحتياط العالمية الرئيسية، والاساس لنصف العملات في العالم، وهو العملة 
الرئيسية بالعالم، فهو يمثل (1 6\%) من حائزات الاحتياطي الرسمي (الجدول 1)، ويستخدم في (85\%) من

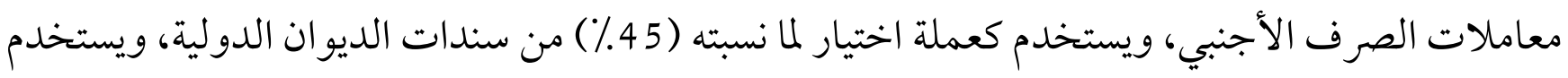
ايضا كفو لأكثر من نصف الصادرات العالمية وهذه ليست ميزة قليلة (Dadush، 2011، 88 ). وقد كان اسناد معظم المعاملات الدولية للدولار لا مفر منه عمليا بعد الحرب العالمية الثانية، عندما كان الناتج الاجمالي للولايات المتحدة يمثل نحو (50٪) من المخرجات العالمية، وعملتها هي العملة الوحيدة القابلة للتحويل عالميا، لكن الانتعاش اللاحق في اوروبا واليابان والذي تبعه نمو سريع في الاسو اق الناشئة واهمها الصين، ادى الى تحول في التركيب الاقليمي في الناتج المحلي الإجمالي، وظهور عدد من العملات القابلة

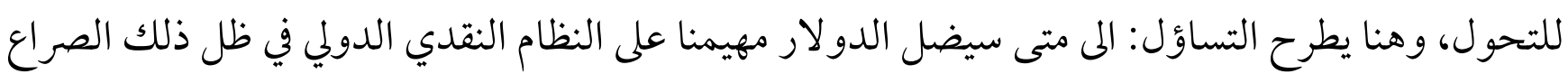
الدائر في اطار الحرب الاقتصادية، ويرى بعض المحللين ان الوضع الدولي الحالي يظل مشابها لما كان قبل 30 عاما، ومن ثم لاتزال الو لايات المتحدة القوة العسكرية العظمى الوحيدة، وقوتها الاقتصادية تفوقها " ويتأكد ذلك بحقيقة ان حكومتها تسيطر على أكبر واغنى سوق من بين اسو اق الاقتصاد العالمي، كما ان سمو شانها الملالي يتأكد من خلال وضعها غير المهدد كمصدر للوسيط الذي لايزال مفضلا في اسو اق الصرف ونس والمعاملات

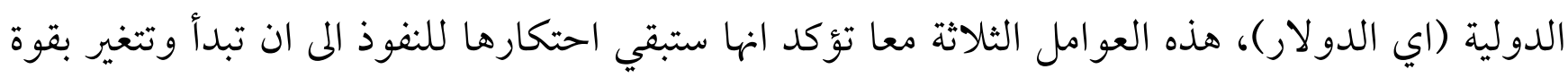
عملية التصويت في النظام النقدي الدولي (Miguel، 2011، 20 20. وقد اكتسبت الصين بعض الحلفاء في الآونة الاخيرة، وتعول بدعم واضح من دول البريك (BRIC) الاخرى:

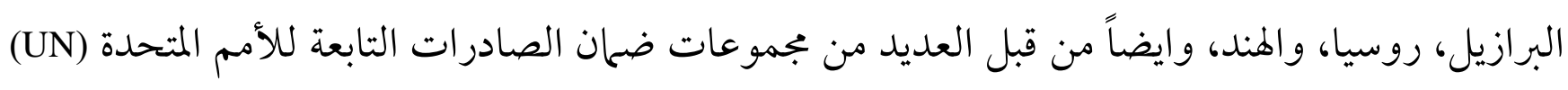
مثل لجنة الخبراء والأنكاد، التي اعلنت جميعها ان النظام الحالي غير مستقر ومن ثم هناك حاجة ماسة لا يجاد حل متعدد الأطراف، والأكثر غرابة، ان تقييا ماثلا صدر حديثا عن صندوق النقد الدولي في 10 20، والذي كان المى وقت قريب جدا لا يجرؤ على التشكيك في معيار (FDS) الدولار المرن (Miguel، ويرى البعض انه وباستبعاد المجال العسكري فان الولايات المتحدة في موقف أضعف نسبيا اليوم عنه في الثخانينات، ولكن ليس بالتأكيد بالأرقام المطلقة، فسوق الاستهلاك الامريكي لا يزال الاكبر جذبا للطلب في 
العالم، ورغم ان الصين الان تصدر أكثر من الاتحاد الاوربي والولايات المتحدة، لكن لا تزال الو لايات المتحدة سوق تصدير اكبر من منطقة اليورو، وفي مجال التمويل، فإن وول ستريت ليست المهيمنة بالتأكيد الآن كما كانت في الثمانينات، فالتكامل الملالي في اوروبا قد قلل المسافات لكنها لاتزال المركز المالي الاكبر اهمية في العالم، والاهم من ذلك، انه لا يوجد سوق سندات مفرد يمكنه منافسة سوق سندات الخزانة الأمريكي، الا انه وحسب ما يرى بعض المحللين فان الولايات المتحدة تخسر على نحو متزايد موقعها القيادي في قيادة اقتصاد السوق العالميأوبالتالي النظام النقدي الدولي الذي يقاد اساسا بقوى السوق، وقد فتحت الازمة المالية الاخيرة التي بدأت في وول ستريت وما نجم عنها من حروب عملة الباب للمناقشات حول بدائل مكنة أكثر تنسيقا و ادارة، واهمها حقوق السحب الخاصة على ان يكون الريمنبي أحد مكوناتها، لاسيها بعد ان اقرت الفكرة لجنة تابعة للأمم المتحدة، وهي الفكرة التي سبق اندعت اليها الصين وطالب بها عدد من خبراء الاقتصاد في العالم في ظل المخاوف المتعلقة بالدولار وبالاقتصاد الأمريكي (عادل، jef1.kcorp.net). وبطبيعة الحال لن تتخلى الولايات المتحدة عن منزلة الدولار المهيمن بسهولة مما يجعل الباب مفتوحا لمزيد من الصراعات والحروب الاقتصادية بين الاقتصادات الكبرى بالعالم والتي ها تأثيرات سلبية على الاقتصاد العالمي عموما و الاقتصادات النامية بوجه خاص.

\section{المحور الثالث: السيناريوهات المستقبلية للحرب الاقتصادي بين أمريكا والصين}

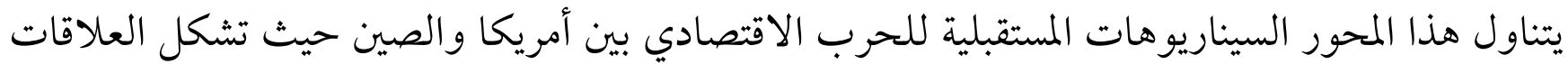
الامريكية الصينية نموذجاً يجمع بين الصراع والتعاون الحذر، حيث تمتلك كل واحدة منها عناصر القوة في ذلك، فأمريكا تحاول الاحتفاظ بالهيمنة على النظام الدولي، والصين بثقلها الاقتصادي والسياسي والعسكري المتز ايد تسعى من أجل الوصول الم قمة النظام الدولي. وان شكل العلاقات الامريكية الصينية في قادم الايام سوف يحدد صورة وملامح النظام العالمي الجلديد، حيث انها علاقات معقدة تتراوح بين التقارب والتصارع من حين لأخر بسبب اختلاف المصالح بينها. (ابراهيم، 2016) 


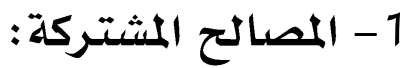

حيث تنظر الولايات المتحدة الامريكية الى الصين نظرة الدولة الناهضة التي لها قوة ودور اقليمي وعالمي، كما ان الصين تنظر الى الولايات المتحدة الامريكية على انها القوة العظمى ذات المصالح المتشعبة على مستوى العالم، كما انها يمكن ان تلعب دوراً مهاً بالنسبة للصين. لذلك فان الو لايات المتحدة الامريكية ترى ان صعود الصين بات يهدد مصالحها وأمنها القومي كذلك فان الصين ترى ضرورة التوجه الى عالم متعدد الاقطاب لا توجد فيه هيمنة لدولة واحدة وهي أمريكا بل هناك توازن بين القوى المختلفة. كحا ان الاهداف الصينية من وراء سياستها الخارجية تسعى ان تكون سوقاً موجهاً للعالم أو مصنع للعالم اضافة الى تحقيق درجة عالية من الصادرات التي تدر اباحاً عليها، حيث عملت على استغلال سوقها الاستراتيجي نحو الخارج، وتهدف من هذا التوسع رفع ناتج الفرد من الناتج الاجمالي القومي وتغيير هيكلها الاقتصادي لتكريس انفتاحها على الاقتصاد العالمي اضافة الى خلق نظام متعدد القوى ومناخ عالمي يتفق مع تطلعاتها. أما الاهداف الامريكية تجاه الصين تتأتى من مصالحها السياسية والاقتصادية والامنية بالقارة الاسيوية خاصة أن المنطقة غنية بالاستثارات التجارية وكونها مورد اقتصادي مهم لما تتمتع به من ثروات طبيعية وقوة تسويقية. كما ان الاستراتيجية الصينية في الاساس تقوم على عامل الزمن ولا تتعجل في مزاحة امريكا في ريادة العالم على اعتبار ان امريكا تتحمل العبء الاكبر في التصدي للمشكلات الدولية، الامر الذي استزف قو اها في ظل التقصير في مجال تنمية نظام عالمي أكثر تعاوناً وأكثر انسجاماً وتعميم النزاعات بدلاً من احتو ائها أو اطفاء نيرانها.

\section{1- تغييير المعادلة:}

وفقاً للتطورات التي حدثت في العالم، فان الولايات المتحدة الامريكية لم ولن تقبل بتغيير المعادلة لصالح دولة غيرها لتكون القوة العظمى الاولى في المنطقة سواءً عسكرياً أو اقتصادياً، حيث تشهد العلاقة بين واشنطن وبكين حالياً موجة من التصعيد السياسي والاستراتيجي خاصة من الجانب الامريكي وعلى الرغم من ذلك فان هذا التصعيد ليس بجديد عن سابقاته خلال الفترة المنصرمة الماضية، لكن من حيث الشكل قد يكون 
الاكثر حدة لكنه لن يصل الى الحرب فكلا الدولتين تحتاجان الم بعضها، حيث بقدر وجود التوترات والخلافات الجوهرية فانه يقابلها وجود مصالح مشتركة. لكن في نفس الوقت تحاول امريكا اتباع سياسة تصعيدية من خلال تطويق الصين على جميع المستويات للحد من قوة اقتصادها المتنامي، على الرغم من ان الصين حريصة على طمأنة العالم أن صعودها لا يهدف الى السيطرة. وان التدهور الذي تشهده العلاقات الامريكية - الصينية على خلفية جائحة فايروس كورونا المستجد و الانتقادات والاتهامات المتبادلة بين مسؤولي البلدين بشأنها ومع اشتداد وتيرة الحرب التجارية بين البلدين والجدل المحموم حول الانفصال عن سلاسل التوريد الصينية ظهرت أزمة فايروس كورونا المستجد التي حسمت الجدل المى ضرورة خلق سلاسل تصنيع وتوريد خارج الصين وانه متى تنتهي أزمة انتشار الفيروس فتسرع الاقتصادات العالمية نحو تنويع سلاسل التوريد والتصنيع لكي لا تبقى معتمدة على الصين بشكل اساسي كما ان هناك عدة شركات امريكية بدأت بالفعل في تنويع سلاسل توريدها والاتجاه نحو فيتنام بدلاً من الصين. (فخري، 200 20.

\section{النتائج:}

1 - تكتسب العلاقات الامريكية الصينية أهمية خاصة، حيث ان هذه العلاقة تمثل مرحلة انتقالية ستؤدي تفاعلاتها في النهاية الى تشكيل معالم النظام العالمي في هذا القرن (الواحد والعشرين) وهذا يرجع الى

$$
\text { الوزن النسبي لكالا البلدين. }
$$

2- ان امريكا تعتبر حتى الان هي القوة العظمى الوحيدة بين بجموعة من القوى الكبرى، والصين بثقلها الديموغر افي و الاقتصادي و العسكري المتنامي تسعى ان تكون قوة عظمى تلعب دوراً بارزاً ومؤثراً في حركة التفاعلات الدولية. 3- تخوض امريكا والصين منافسة طويلة على الصدارة الاقتصادية، حيث ان النمو الاقتصادي للصين أسرع من النمو الاقتصادي الامريكي، وبالتالي فانه قد يجعل الصين تصل لمرحلة التكافؤ مع الولايات 
المتحدة الامريكية وقد تحل محلها لتصبح القوة المهيمنة في النظام الدولي مع تزايد الناتج القومي الا جمالي وتضخم عدد سكانها.

4- ان ظاهرة صراع الحرب الاقتصادية تقودها الاقتصادات الكبرى في العالم بغرض تحقيق ميزة تنافسية لصادر اتها على حساب شر كائها بالتجارة العالمية.

5- من الطبيعي ان تقوم الاقتصادات الكبرى المتضررة برد مماثل او اتباع سياسات حماءيه متشددة وهو ما يؤدي حتما الى تهديد الاستقر ار الاقتصادي العالمي برمته وذلك بشقية النقدي والحقيقي.

6- ستكون الدول النامية هي الخاسر الاكبر من ذلك الصراع خاصة تلك الدول التي لا تزال تربط عملتها بالدو لار الامريكي الذي يعد القائد الأساسي لذلك الصراع. 7 - ان معظم الدول وخاصة العربية منها تعتمد في اير اداتها بشكل اساسي على صادراتها من السلع الاولية المقومة بالدولار واهمها النفط وجزء منها على الاستثمارات الخارجية والمقوم معظمها ايضا بالدولار، فضلا عن ان الدولار الامريكي يشكل النسبة الاكبر من احتياطات تلك الدول، من هنا يتضح حجم الأثر الذي يتركه انخفاض قيمة الدولار الامريكي في أطار الحرب الاقتصادية على اقتصاديات تلك

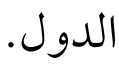

التوصيات:

استنادا المى اهم النتائج التي تم التوصيل اليها في هذا البحث يمكن الخروج ببعض التوصيات فيما يتعلق بما يجب على الدول النامية بشكل عام والدول العربية بشكل خاص القيام به للتقليل من تداعيات ذلك الصراع على اقتصاداتها من خلال ما يأتي: 1 - على الدول النامية ان تفك ارتباط عملاتها بالدو لار الامريكي وربطها بسلة من عملات اهم شركائها التجاريين، لتقليل مخاطر تقلبات اسعار الصرف. 
2- تنويع احتياطاتها الأجنبية لتكون مكونة من ذات السلة اضافة الى الذهب وذلك لتقليل من آثار التقلبات في قيمة الدولار على اقتصاداتها.

$$
\text { 3- تنويع الاستثمارات من حيث العملات موضوع الاستثمار و كذلك جغرافنيها. }
$$

4- ضرورة التنسيق فيما بين الدول النامية وتوحيد مو اقفها في كافة القضايا حتى يكون لها وزن نسبي كبير يؤخذ بعين الاعتبار في كافة المحافل الدولية بها يضمن قوة وفعالية التفاوض في كافة القضايا الدولية وان تكون شريك اساسي في تقرير السياسيات الاقتصادية المتعلقة بتلك القضايا. 5- يجب على الدول العربية اضافة الى ما سبق ان تشرع بشكل جاد ومسؤول بتنفيذ كل الاتفاقيات والمعاهدات الخاصة بالتكامل الاقتصادي العربي والذي أصبح ضرورة حتمية املتها الظروف والاوضاع الاقتصادية العالمية المعاصرة، الامر الذي يمكنها من تقليص ارتباطها بالخارج ومن ثم تجنب تأثرها بتلك الاوضاع وما ينجم عنها من تبعات الى ادني حد ممكن وذلك من خلال قناتين رئيسيتين: أ. تنويع قاعدة الانتاج حيث يمكنها من خلال التكامل الاقتصادي القيام بالاستثارات العربية البينية في كافة المجالات بها فيها المجال الصناعي والزراعي مستفيدة من وفرة الموارد الطبيعية وتنوعها واتساع السوق وتميز موقعها الجغرافي الذي يتوسط قارات العالم وبذلك يمكنها من خلال التجارة البينة المركنة الى قاعدة انتاجية عريضة تقليص اعتمادها على الخارج في توفير الكثير من المنتجات.

ب. كذلك ومن خلال تنويع القاعدة الانتاجية يمكن للدول العربية ان تنوع هيكل صادراتها ومن ثم تقليل اعتمادها على الايرادات المتأنية من الموارد الطبيعية التي تعتمد بالأساس على الطلب الخارجي المتذبذب فضلا عن كونها مو ارد ناضبة. 


\section{المراجحع: (1)

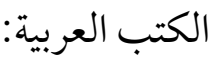

1 ـ دانياليز، جوزيف، وديفيد فانهور، اقتصاديات النقود والتمويل الدولي (مترجم)، دار المريخ للنشر، المملكة العربية

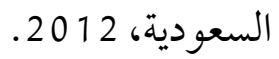

2. ستانليك، مقدمة في الاقتصاد الكلي (مترجم)، منشورات جامعة قاريونس، ليبيا، 1992. 3. كربانين، موردخاي، الاقتصاد الدولي مدخل السياسات (مترجم)، دار المريخ للنشر، العربية السعودية، 2010. مقالات على شبكة المعلو مات الدولية:

4. ابراهيم، ايهان عبد الله عبد الخالق، أثر العلاقات الصينية الامريكية على النظام الدولي منذ عام 2001، رسالة ماجستير، المركز الديمقر اطي العربي، 2016.

5. اقتصاديون، استثمارات العملة الواحدة خطر يهدد ثروات السعوديين في الخارج، الاقتصادية الالكترونية، العدد

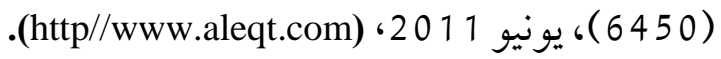
6. بشير، شريق، أثر انخفاض الدولار على الاقتصاد العالمي، اكتوبر 2004، متاح على الموقع

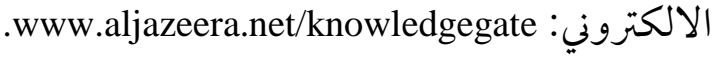

7. بن غيث، ناصر، الحرب الاقتصادية باتت وشيكة، بجلة آفاق المستقبل، العدد (99)، يناير/فبراير 2011، (www.ecssr.com)

8. جلال، محمد سعيد، القصة كاملة: صناعة الحرب الاقتصادية والدين الامريكي وطباعة الدولارات الامريكية (Www.indexsignal.com)، (المجانية، مارس 2013) 9. جلال، محمد، الحرب الاقتصادية وصراع البقاء، 2020)، (www.ecpulse.com). 10. .(www.article.net) $62014 / 3 / 12$ 11 . 1 الحرب الاقتصادية تقوض نمو الاقتصاد العالمي، (www.masralarabia.com). 12. .(www.w- ib.com 
13. زيدان، عصام، الحرب الاقتصادية كارثة على اقتصاديات الدول العربية، 2011، متاح على الموقع الالكتروني:

.(main.islammessage.com)

14 ـ السلماوي، مصطفي، الحرب الاقتصادية اندلعت من جديد و الضحايا اسواق الدول الناشئة، 2013. 15. ضمد، جليل شيعان، الحرب الاقتصادية والنظام النقدي الدولي، الحوار المتمدن، العدد (1 363 )، فبراير 2012،

متاح على الموقع الالكتروني: (www.ahewar.org) .

16. طلب، جمال الدين، الحرب الاقتصادية العالمية قد تطال شظاياها منطقة العربية بشكل موجع، متاح على الموقع

الالكتروني: (www.alrakoba.net).

17 . عادل، محسن، الاقتصاد العالمي 2020، الحرب الاقتصادية المسمار الاخير في نعش النظام النقدي العالمي، متاح على

الموقع الالكتروني: (jef.kcorp.net).

18. العبود، عبد الامير رحيمة، الحرب الاقتصادية: مظاهرها وتطورها في ظل التحولات في النظام النقدي الدولي، متاح

على الموقع الالكتروني: (www.aliraqi.Org).

19. .2020

20 . 2 فرنسا و الصين تتزعمان ماة لإقصاء الدو لار عن الاحتياط، (Www.hawamer.com).

1 2. الفقيه، علي، الحرب الاقتصادية واستمرار الاقتصادات الكبيرة في الازمات، صحيفة اخبا اليوم، ديسمبر 2011. 22. كور، مارتن، الحرب الاقتصادية تعمق الفجوة بين اقتصادات الدول المتقدمة والاقتصادات الناشئة سريعة النمو،

.(2012director@ @ southcentre.org)،2012

محمود، هشام، تقلبات اسعار الصرف تنذر بـ " حرب عملات " في العالم، الاقتصادية الالكترونية، مايو 2013، .23

.(www.aleqt.com/2013/05/18)

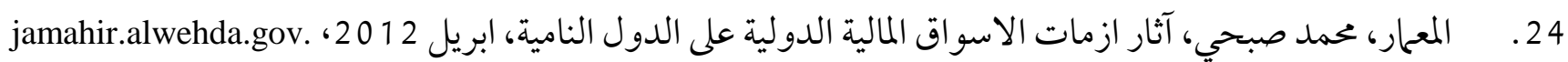
25. مين شين بيه، اعادة تقويم العملة الصينية، هل تنقذ الاقتصاد العالمي؟، يونيو 2010، متاح على الموقع الالكتروني:

(www.shorouknews. Com) 26. مين شين، الحرب الاقتصادية بين الصين وأمريكا، اكتوبر 2010، متاح على الموقع الالكتروني: (www.shorouknews.com) 
27. هل ستزيد الصين احتياطاتها من الذهب؟، الصين اليومية، 11 مايو، 2009، متاح على الموقع الالكتروني:

.(www.ecssr.com)

$$
\text { المراجع الأجنبية. }
$$

Badush, Uri, Vera Eidelman, Currency Wars Carnegie Endowment International Peace, 2011.

Miguel Otero-Iglesias, Currency Wars Between the US and China: Where Does the EU Stand? April 2011.

Murphy, Melissa, Wen Jin Yuan, Is China Ready to Challenge the Dollar, CSIS, October 2009.

Wyplosz, Charles, Currency Wars, European, Parliament, Directorate General for International Policies, Economic and Monetary Affairs, Novamber 2010. 